\title{
Surgimiento de la Escuela de Obstetricia en el mapa social de Costa Rica: finales del siglo XIX y principios del $\mathrm{XX}^{1}$
}

\author{
Daniel Martínez Esquivel ${ }^{2}$ \\ María de los Angeles Meza Benavides ${ }^{3}$
}

Institución: Universidad de Costa Rica

\section{COMO CITAR}

Martínez, D. y Meza, M. (2015). Surgimiento de la Escuela de Obstetricia en el mapa social de Costa Rica: finales del siglo XIX y principios del XX. Rev. Enfermería Actual en Costa Rica, 28, 1-12. DOI: http://dx.doi.org/10.15517/revenf.v0i28.17202

\section{RESUMEN}

Introducción. El presente artículo muestra los resultados preliminares que forman parte de la investigación "Funciones sociales de la Enfermería en los diversos procesos históricos desde la construcción de la Salud Pública en la Costa Rica contemporánea", cuyo propósito es analizar los hechos sociales e históricos que favorecieron la profesionalización de la Enfermería, en un primer momento, con la fundación de la Escuela de Obstetricia en 1897.

Materiales y método: Este artículo responde a una investigación histórica basada en tres pasos: la revisión de fuentes primarias como la Gaceta Médica y los informes de la Junta de Caridad, y fuentes secundarias; la evaluación crítica de los datos; y la interpretación y conclusiones de los hechos.

Resultados: Los hechos que favorecieron la fundación de la Escuela de Obstetricia se relacionaron con la creciente tasa de mortalidad infantil, la necesidad de institucionalizar las prácticas sanitarias e higienizar a la población para construir un Estado fuerte que promoviera el desarrollo económico, así como con la lucha que emprendió la institución médica contra las prácticas populares de atención de salud.

Conclusión. Se concluye que la represión de la curandería y la partería estuvo relacionada con el poder patriarcal. Además, la fundación de la Escuela de Obstetricia se relacionó a las condiciones sociales, políticas y económicas de la época basadas en la línea de desarrollo que estaba teniendo el país.

Palabras clave: enfermería, historia, obstetricia, parteras, salud.

\footnotetext{
${ }^{1}$ Fecha de recepción: 15 de julio del 2014

Fecha de aceptación: 23 de octubre del 2014

${ }^{2}$ Enfermero. Escuela de Enfermería. Universidad de Costa Rica. Costa Rica. Correo electrónico: dmartinez@gmail.com

${ }^{3}$ Enfermera. Escuela de Enfermería. Universidad de Costa Rica. Correo electrónico: mmezab@gmail.com
} 


\title{
Emergence of the School of Obstetrics at the social map of Costa Rica: end of 19th century and beginning of the $20 \mathrm{th}^{1}$
}

\author{
Institution: University of Costa Rica
}

\author{
Daniel Martínez Esquivel ${ }^{2}$ \\ María de los Angeles Meza Benavides ${ }^{3}$
}

\section{CITED:}

Martínez, D. y Meza, M. (2015). Emergence of the School of Obstetrics at the social map of Costa Rica: end of $19^{\text {th }}$ century and beginning of the $20^{\text {th }}$. Rev. Enfermería Actual en Costa Rica, 28, 1-12. DOI: http://dx.doi.org/10.15517/revenf.v0i28.17202

\begin{abstract}
Introduction: This article shows the preliminary results which are part of research "Social functions of nursing in different historical processes since the construction of the public health in the contemporary Costa Rica", whose purpose is to analyze the social and historical facts that favored the professionalization of nursing, initially, with the Foundation of the school of obstetrics in 1897.

Method: This article responds to a historical research based on three steps: the revision of primary sources such as the medical Gazette and the reports of the Board of charity, and secondary sources; the critical evaluation of data; and interpretation and conclusions from the facts.

Results: The facts that favored the Foundation of the school of obstetrics was linked with the rising rate of infant mortality, the need to institutionalize health practices and sanitize the population to build a strong State for promoting economic development, as well as the struggle undertaken by the medical institution against popular health care practices.

Conclusion: It is concluded that the repression of the quackery and midwifery was related to patriarchal power. In addition, the Foundation of the school of obstetrics was related to social, political and economic conditions of the time based on the line of development which was taking the country.
\end{abstract}

Key words: health, history, midwife, nursing, obstetric.

\footnotetext{
${ }^{1}$ Date of receipt: July 15, 2014

Date of acceptance: October 23, 2014

${ }^{2}$ Nurse. School of Nursing. University of Costa Rica. Costa Rica. E-mail: dmartinez@gmail.com

${ }^{3}$ Nurse. School of Nursing. University of Costa Rica. E-mail: mmezab@gmail.com
} 


\section{.}

\section{INTRODUCCIÓN}

La historia de la Enfermería se ha construido a partir de otras disciplinas como la Historia, la Medicina o la Sociología, las cuales enaltecen lo religioso, la sumisión y lo profano, elementos que dificultan la visualización de la mujer en el ámbito de la salud (Coelho, Susskind, Ballesteros, 2008). Por este motivo, es importante desarrollar una construcción sociohistórica de la Enfermería a partir de una cosmovisión propia con la que se construya un significado que permita comprender el contexto en el que se desarrolla y la importancia que tiene la profesión para la población costarricense.

Considerando lo anterior, el presente artículo tiene como objetivo el estudio de las condiciones sociales, políticas y económicas que influyeron en la constitución de la primera Escuela de Obstetricia en Costa Rica y muestra los resultados preliminares que forman parte de la investigación "Funciones sociales de la enfermería en los diversos procesos históricos desde la construcción de la Salud Pública en la Costa Rica contemporánea". En este estudio, participan investigadores de las áreas de Historia y de Enfermería, quienes con una visión transdisciplinaria analizan los fenómenos sociales para conocer y comprender la participación de la Enfermería en el desarrollo del Estado costarricense. El propósito final de la investigación es conmemorar el centenario de la fundación de la Escuela de Enfermería en Costa Rica, en el año 2017.

A partir de la investigación sobre las condiciones en que se funda la Escuela de Obstetricia, se formula las siguientes interrogantes: ¿Cuáles fueron las condiciones sociales, políticas y económicas de la población costarricense a finales del siglo XIX y principios del siglo XX? Y, ¿de qué manera la fundación de una Escuela de Obstetricia se constituyó en una respuesta de atención a esta población?

\section{MATERIALES Y MÉTODO}

Al ser este estudio una investigación histórica, se caracteriza por analizar las transformaciones sociales sucedidas, en este caso, en Costa Rica a finales del siglo XIX y principios del siglo XX, sobre las cuales se funda la Escuela de Obstetricia y se responde a las interrogantes planteadas, proceso para el se siguió tres pasos: recolección de datos, evaluación crítica de los datos y presentación y conclusiones de los hechos (Coelho et al, 2008).

Luego, se buscó fuentes históricas con la finalidad de delimitar periodos y personajes clave en la construcción de la Salud Pública, en cuanto a lo general, y de la Enfermería, en lo particular. Dentro de esta búsqueda se incluyó fuentes primarias como varios volúmenes de la Revista Gaceta Médica, incluyendo la de 1896, en los que aparecen las primeras anotaciones sobre la importancia que tendría para la población costarricense la apertura de un centro de enseñanza sanitaria. También, se consultó los informes de la Junta de Caridad y varias fuentes secundarias relacionadas con el tema.

Además de contar con una descripción de la sociedad costarricense de la época en estudio, se inició la formulación de lo que se denomina la red social de actores y estructuras, a quienes se dará seguimiento en futuros estudios sobre el tema. 
Después de dicho proceso, se plantea dos categorías de análisis: las condiciones sociales de Costa Rica de finales del siglo XIX y principios del siglo XX y la fundación de la Escuela de Obstetricia.

\section{Consideraciones éticas}

En el desarrollo de esta investigación histórica no participaron seres humanos, dado que las fuentes consultadas son primarias. Sin embargo, se incluyó ciertas consideraciones éticas que garantizarán el buen desarrollo de esta investigación, como por ejemplo se respetó la autonomía de los investigadores en cuanto a la interpretación de los datos recolectados, quienes son personas conocedoras de la temática y con responsabilidad histórica.

En cuanto a sus ventajas, se espera producir un beneficio para la Enfermería en cuanto a comprender sus funciones sociales a través de la construcción histórica en Costa Rica ; entre otras funciones, se pretende obtener resultados fructíferos para el bien de la sociedad y, específicamente, el desarrollo de la Enfermería en Costa Rica.

Toda la información obtenida se manejó con la objetividad posible, procurando no lesionar a aquellas personas que tengan una opinión diferente.

\section{RESULTADOS}

En este apartado, se presenta los resultados de la revisión de fuentes primarias que permitieron un acercamiento a la problemática planteada para responder a las interrogantes y objetivo del presente artículo, razón por la que es imprescindible mostrar los fragmentos de esas fuentes que apoyan tal idea.

El contexto en el que se desarrolla la Escuela de Obstetricia en Costa Rica se da en una época de gran auge de la medicina, acompañada de la curandería y la partería, lo cual supone una lucha ideológica, política y social por definir las prácticas más adecuadas para atender la salud de la población.

Para finales del siglo XIX, y en parte como medida para luchar contra tal situación, surgió la Facultad de Medicina, Cirugía y Farmacia en sustitución del Protomedicato que funcionaba desde hacía años en Costa Rica y que fue perdiendo poder debido a su ineficaz trabajo para suprimir el abuso de los curanderos en actividades consideradas como delito. Respecto de lo anterior, Lachner (1902) menciona que

El 29 de agosto de 1895 se transformó el Protomedicato en Facultad de Medicina, Cirugía y Farmacia, a la cual están sujetos los médicos, farmacéuticos, dentistas y obstetrices, debiendo tener un carácter oficial docente y consultivo. Este cuerpo resolvió por de pronto empezar a publicar un órgano mensual, científico médico, lo que se llevó a cabo apareciendo el $1^{\circ}$. de mayo de 1896 el primer número de la "Gaceta Médica de Costa Rica” (p.41).

Aunado a este enfrentamiento científico-político, las condiciones sociales no eran nada favorables y la población costarricense vivía en un estado precario, lo que se describe en dos casos concretos que se reseñan en la Gaceta Médica de 1896; sobre el primero se menciona: "El 9 de abril fui llamado a casa de la señora X, quien, 


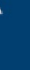

según me dijo la partera que la asistía, estaba en trabajo hacía dos días, habiéndosele roto espontáneamente la fuente desde 24 horas antes" (Soto-Alfaro, 1896, p.5). En cuanto al segundo se narra lo siguiente:

La parturienta había dado a luz hacía dos horas a un hermoso niño de ocho libras de peso que produjo una gran desgarradura incompleta del periné. Media hora después debido a inhábiles e intempestivas tracciones de una buena vecina que fungía de comadrona, se rompió el cordón, hubo desprendimiento parcial de placenta y como consecuencia se produjo una hemorragia grave que con justificado motivo alarmó a la familia" (Ros-Pochet, 1896, p.51).

A través de dichos relatos se describe las condiciones en que se atendía el parto en las poblaciones pobres en las que las mujeres no tenían acceso a la atención prenatal ni durante el parto, además, como en otras descripciones que aparecen en esta misma revista, la atención se brindaba en las casas y el médico acudía cuando el parto se complicaba. También, es importante señalar la capacidad de la partera para atender el parto, identificar la emergencia y acudir al médico; no obstante, ese panorama fue el que propició la fundación de la Escuela de Obstetricia, considerada una acción política prioritaria.

En la Revista Gaceta Médica del 1 de agosto de 1896, se alude a la necesidad de establecer una Escuela de Obstetricia. Entre los pormenores a los que hace referencia el médico César Borja, en ese momento director de la revista, para justificar el establecimiento de dicha escuela se cita la alta mortalidad infantil y la dificultad de contar con estadísticas confiables sobre las causas de deceso, la falta absoluta de cuidados médicos que necesitan la madre y el recién nacido, y los cuidados higiénicos que no pueden dar las madres pobres, porque no cuentan con los medios ni la instrucción necesaria, además; además, hay que mencionar que tales madres no podían contar con la asistencia de un médico de cabecera que les brindara atención en el parto, lo cual justificaba la muerte de muchos niños.

Por otra parte, dicho médico agrega que ciertas acciones eran realizadas por personas sin preparación, cuya participación se relacionó con la muerte por tétanos; no obstante, dicha mortalidad infantil podría haberse evitado si los partos hubieran sido atendidos en una maternidad u hospital para parturientas; a pesar de ello, más adelante, con la ayuda de otra institución muy fácil de establecer, la Escuela de Obstetricia, se logró resultados más que significativos, situación a la que se refiere Borja (1896) en las siguiente líneas:

Un establecimiento de esta naturaleza les abriría a muchas mujeres pobres las puertas de un porvenir seguro y honrado, antes desconocidos para ellas: cuántas niñas sin patrimonio y sin amparo que hoy tienen que luchar por la vida entre los dos caminos terribles de la prostitución y la miseria, no hallarían en dicha escuela un verdadero asilo de instrucción y moral por el momento y un punto de partida para el trabajo honrado y productivo después (p.112).

Al respecto, en el Informe de la Junta de Caridad (1896) se señalaba lo siguiente: "las mujeres aquí trabajan mucho y son muy mal pagadas; y más en las ciudades, como sirvientas, pues eso es causa fundamental para que tantas se introduzcan a la vida libre y a la venta de su vergüenza" (p.6). Tal comentario se refiere a la importancia de la escuela en cuanto a brindar diferentes oportunidades a las mujeres en cuanto a tener un mejor desarrollo social y económico. 
De acuerdo con lo anterior, la Escuela de Obstetricia tendría dos misiones principales: por una parte, brindar a muchas mujeres pobres, de vida licenciosa o mujeres prostitutas, la oportunidad de estudiar y convertirse en obstétricas, y por otra, velar por la salud de las mujeres en el parto, lo cual queda corroborado en las palabras de Borja (1896): "Y estas niñas, convertidas más tarde en profesoras instruidas, circunspectas y caritativas, cuántos bienes no harían no solo en la sociedad sino también en las gentes más infelices del pueblo" ( p.112). Según el autor, "estas gentes" son las mujeres pobres del país que no pueden tener una atención médica, ya que es costosa, y solo pueden contar con la caridad.

Continuando con el recuento histórico, se debe señalar que previo a la fundación de la Escuela de Obstetricia, la Directiva de la Escuela de Enfermería del Hospital John Hopkings en Baltimore, concedió una beca de estudio para una señorita o viuda que deseara estudiar Enfermería, la cual fue adjudicada a Emilia Rivas V. de Santos de parte de la Facultad de Medicina, Cirugía y Farmacia que la financió con una ayuda económica (Gaceta Médica, 1897). Este hecho significó una antesala para concretar el plan de la profesionalización de la obstetricia y la Enfermería que se venía desarrollando en el país y demuestra el interés de los sectores político-sanitarios para que el desarrollo de la escuela fuera un éxito.

Rucavado y Ross (1900) presentaron el reglamento que sentó las bases de la primera Escuela de Parteras y de la primera maternidad. En su discurso, Rucavado (1900) menciona que

la beneficencia pública necesitaba de una casa de maternidad que salvara a tantas madres de dar a luz en la miseria y arrancara de una muerte segura a los recién nacidos; y la necesitaban las señoras dedicadas a la obstetricia como cumple el estudio clínico del arte de los partos (p. 71).

Instaurado el servicio de maternidad, la Facultad de Medicina, Cirugía y Farmacia (1900) se pronunció a través de la Revista Gaceta Médica de la siguiente manera:

Ya no morirá el niño privado de los auxilios de la ciencia. Ya no se verá el triste espectáculo del alumbramiento en el escenario del cuartucho privado de luz, desprovisto de los elementos más necesarios a la higiene de ese grandioso momento en la vida de la mujer, ya no se verá más la falaz fisonomía de la comadrona empírica, casada clandestinamente con la ciencia, pero enamorada de las tradiciones nefastas, enemiga del agua, del aseo y de todo lo que como en vida que es, busca a la vida (p.70).

La cita anterior ejemplifica la lucha de poder que existía entre la profesión médica y cualquier otra forma de intervención que se le pareciera, mas sin los lineamientos del caso.

En cuanto a las políticas de la Escuela de Obstetricia, la administración fue independiente, con un reglamento específico que fue redactado entre la Facultad de Medicina, Cirugía y Farmacia y la Junta de Caridad. Se menciona además, las posibilidades de contar con un lugar en el que las estudiantes puedan desarrollar sus prácticas, dado que "lo era menester organizar bien desde el principio, la Clínica de Partos, y para ello no hay necesidad de emprender ninguna obra extraordinaria, ni hacer ningún sacrificio” (Borja, 1896, p.113). 
.

\section{DISCUSIÓN}

\section{Condiciones sociales de la Costa Rica de finales del siglo XIX y principios del XX}

Las condiciones sociohistóricas de Costa Rica a finales del siglo XIX y principios del siglo XX definen lo que se podría llamar una primera etapa de la historia social de la Enfermería, ya que contextualizan el momento en que se establece la Escuela de Obstetricia, por lo tanto, es preciso referirse a este período en el que Molina y Palmer (2004), destacan dos hechos importantes: la presencia de la cultura escrita con el desarrollo de la imprenta y con ella la alfabetización, y el desarrollo en el tema de la salud.

Para esta época, Costa Rica era un país cuya economía se sustentaba en la exportación del café, lo cual favoreció la incorporación de la economía al mercado internacional, alrededor del que se unió un fuerte sector de cafetaleros y comerciantes que controlaron la producción, la riqueza y el aparato Estatal (Salas-Víquez, 1987; Gudmundson-Kristjanson, 1993). En este sentido, el país, principalmente la capital San José, era "próspero" pero con grandes contradicciones sociales: por un lado, gran mayoría de la población vivían en extrema pobreza, sin posibilidades de atención médica y sin educación, por el otro, existía una élite cafetalera y un tercer grupo integrado por artesanos, algunos letrados y profesionistas principalmente extranjeros que se ubicaban en la medicina, el derecho y la educación (Samper-Kutschbach, 1979).

Una característica importante de la Costa Rica de esta época es la influencia de los inmigrantes de Europa, Estados Unidos y América Latina, entre los que se encontraban empresarios, intelectuales y científicos (HerreraBalharry, 1985), variedad alrededor de la que se construyó una Costa Rica polarizada, aunque primordialmente campesina, como se describe en el siguiente fragmento "el paisaje que San José le ofrecía a don Avelino era muy distinto: un casco urbano diminuto, preso entre montañas y asediado por miles de cafetos (Molina-Jiménez et al., 2004, p.23).

Dentro de un escenario tan variado, abundaban las contradicciones; por ejemplo, a pesar de que la educación en su estructura contaba con grandes deficiencias, se logró avanzar en alfabetización, especialmente en el centro y áreas cercanas a la capital. Los censos poblacionales señalan que para 1892 solo el 8\% de la población asistía a la escuela, ya en 1927, más del 85\% de los costarricenses mayores de 9 años estaban alfabetizados en las ciudades principales, un $66 \%$ en el campo y, en general, se contaba con un $59 \%$ en todo el país, a excepción de las mujeres que presentaban cifras más bajas (Molina et al, 2004).

Sin embargo, las condiciones de pobreza de una parte muy importante de la población se reflejaban en un estado precario de la salud y la posibilidad de contar con atención médica era casi nula. Al respecto, Lanning, citado por Molina et al (2004), menciona que para 1821, año en que Costa Rica se independizó, no se contaba con un solo médico en la provincia, la atención a la salud se daba en las familias y se recurría a los servicios de empíricos y curanderos urbanos y rurales; sobre el tema, González (2005) enfatiza que para la época las condiciones de salud eran deficientes. El acceso a los servicios de salud no era equitativo y la mayoría de enfermos recibían cuidados de personas sin conocimientos científicos.

En cuanto a la curandería en Costa Rica, Molina et al (2004), señalan que a pesar de ser un país pequeño y pobre, su influencia de fue muy dinámica y variada. Hubo una fuerte contradicción entre esta y el ejercicio de la 


\section{.}
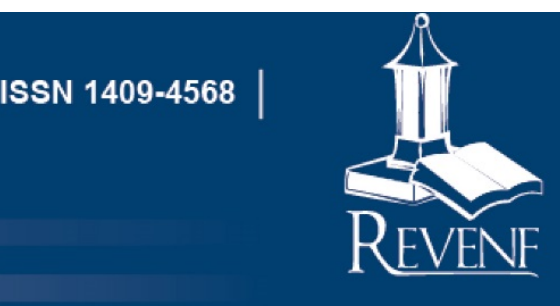

\section{Revista Electrónica Enfermeria Actual de costa Rica}

www.revenf.ucr.ac.cr

medicina formal; mientras que la primera se convertía en una vía que conectaba lo moderno con lo tradicional, lo lícito con lo ilícito y lo racional con lo irracional, la segunda ejercía su influencia política para defender su poder social y económico, motivo por el que se convirtió en la principal fuerza de persecución de estas formas de atención a la salud. Aparte, de la incipiente profesión médica, las autoridades judiciales y eclesiásticas se unieron a la persecución; Marín (2000) considera que en el periodo entre 1800 y 1949 se gestó y consolidó un fuerte aparato de control social para normar las prácticas populares de curación, razón por la que, según Marín, los argumentos de la ciencia sanitaria, se filtraron en la ideología dominante para potenciar el poder de la racionalidad científica, cuyo fin es regular las prácticas sociales alrededor de lo que él llama la ideología terapéutica liberal (Marín, 2000, p.4).

Desde la ideología liberal, la necesidad de consolidar el Estado Nación requería mantener a la población alfabetizada y sana, de manera que se aseguraba las condiciones para el desarrollo económico. El progreso capitalista, asentado principalmente en la capital, "exigía convertir a campesinos y artesanos en ciudadanos saludables, higiénicos, instruidos, patriotas, respetuosos de la ley y fieles a la ideología liberal" (Molina et al, 2004, p.33). Dicha ideología era defendida por los grupos que ostentaban el poder político y económico del país, encontraba entre sus filas a los más reconocidos médicos quienes, en su gran mayoría, además de contar con prestigio social, desempeñaban puestos públicos que les aseguraba una participación directa en las políticas estatales.

Como parte de esta política de Estado, en 1826, se intentó fundar un hospital en San José durante el gobierno de Juan Mora Fernández (1824-1825, 1825-1829, 1829-1833), mas no es hasta 1845 cuando se emitió un decreto impulsado por el diputado José María Castro Madriz (1844-1846), para la creación del Hospital San Juan de Dios. En tal ocasión, Castro Madriz justificó sus acciones manifestando que

la imperiosa necesidad de abrir a la ciencia médica, apenas naciente en nuestro suelo, un campo de elevación y progreso y un refugio a la humanidad doliente, son razones que no se ocultan a la sabia penetración de los Representantes del Pueblo, para hallarse tan impulsados como yo, a la erección de un Hospital General del Estado (González, 2005, p.175).

A partir de lo anterior es correcto afirmar que a pesar de las políticas económicas del momento, la salud se ha asociado con un discurso humanista y caritativo. Sin embargo, Rosenberg (1983), considera que la fundación del hospital obedeció a un reconocimiento de la problemática social existente en la que la anquilostomiasis, la malaria y la fiebre amarilla amenazaban a la población que tenía como único recurso de atención la caridad privada. Según este autor, para ejercer mayor control sanitario, en 1857 se fundó el Protomedicato de la República con el propósito de atender todos los asuntos relacionados con la higiene y salud pública en lo que respecta la incorporación y la disciplina de los médicos, dentistas y farmacéuticos.

En este contexto, cabe señalar que con la creación del hospital, comenzó la participación de la mujer en el área de la salud formal. En 1864, se constituyó un grupo de apoyo de mujeres en las labores del hospital, integrado por Ignacia Sáenz de Gallegos, Esmeralda Oreamuno de Jiménez, Elena Giralt de Ulloa, María Montealegre, Manuela Gallegos, Mariana Devars, Feliciana Quirós de Bonnefil y Juana Fernández (González, 2005). Además, la presencia de mujeres curanderas se asoció al oficio de parteras, traídas también del extranjero para atender a la mujeres acaudaladas, razón por la que se les consideró mujeres benefactoras del pueblo. 
Lejos de reconocer la participación social de la mujer en los ámbitos formal e informal, pareciera que estos hechos generaron una lucha entre la medicina formal y la medicina popular, lucha en la que la mujer tuvo un papel silencioso, ya fuera como comadrona o curandera que, en el plano de la atención formal, fue nulo. En esta disputa, el hombre fue el eje, debido a que en el caso de curanderos con algún poder económico y prestigio no eran perseguidos, ni tampoco su principal clientela: las mujeres. En el plano de la medicina formal no se documenta la presencia de mujeres, al menos en la época de los primeros años del siglo XX: esta diferenciación de clase y de género, permite explicar una parte importante de las funciones sociales de la Enfermería en este momento histórico, razón por la se plantea a continuación las siguientes interrogantes:

¿Cómo la constitución de una Escuela de Obstetricia ayudó a resolver los problemas de salud de la época, hasta convertirse en una estructura más de control social que permitió afianzar aún más a la medicina formal en la lucha contra la curandería y las prácticas populares?

¿Qué papel tuvieron las mujeres durante este momento histórico de la salud en Costa Rica?

Estas interrogantes guiarán el trabajo conforme avance la investigación, mas no serán respondidas en este artículo.

\section{Condiciones sociales y establecimiento de la Escuela de Obstetricia}

Como ha sido señalado, el ejercicio informal de la partería en la sociedad costarricense supuso una contradicción para el desarrollo que estaba teniendo la ciencia médica, incluso, esta práctica era relacionada a ideas mágico-religiosas como exponen Molina et al (2004) y Marín (2000), "la posibilidad de un traslape con la brujería es evidente, lo mismo que con un catolicismo sincrético cargado de supersticiones" (Molina, 2004, p.143).

En dicha época, la realidad de la mujer tenía rostro de pobreza y las posibilidades de trabajo eran nulas (Ramírez, 1985), por lo que muchas debían optar por prácticas de riesgo, como la prostitución, para poder subsistir. Además, sus oportunidades de desarrollo eran limitadas.

A pesar del contexto, la creación de la Escuela de Obstetricia coincidió con la fundación del Colegio Superior de Señoritas, en 1888, y con él una sección para educación normal, lo que podría señalarse como un progreso en la incorporación de la mujer al trabajo, sin embargo, según Malavassi (2002), no fue más que la profesionalización de las labores domésticas, la de educadora y la de cuidadora de la salud, mas no se incluyó su presencia en la vida pública.

La iniciativa de Borja de fundar la Escuela de Obstetricia fue asumida por los médicos Genaro Rucavado y Ross Pochet con el respaldo de Carlos Durán y la Facultad de Medicina, Cirugía y Farmacia. Finalmente, en 1899 se autoriza la creación de la Escuela de Obstetricia y en el año 1902 se graduaron las primeras obstétricas, Francisca Durán, Elena Echeverría y Adelaida Paniagua (González, 2005).

Se debe señalar que, aún con la fundación de esta casa de enseñanza, la partería continuó en el seno de la lucha entre dos prácticas la ejercida por mujeres, cuyo conocimiento había sido heredado por transmisión oral de sus predecesoras, y la de una obstetricia académica impartida generalmente por médicos, en una de las primeras instituciones de educación en salud que se fundó en Costa Rica (Malavassi, 2002). A partir de ese momento, se 
contó con una educación de varios años de duración, durante los que se impartía materias de asepsia, fisiología, neonatología, entre otras; el plan de estudios estaba fundamentado en los últimos descubrimientos científicos de la revolución bacteriológica, cuya teoría representó una importante herramienta para el poder político que necesitaba legitimar el conocimiento médico científico sobre el saber popular.

Luego, se inauguró la Maternidad del Hospital San Juan de Dios en 1900, patrocinada por la Facultad de Medicina, Cirugía y Farmacia con el apoyo de Jaime Bennet, Elías Tournon, Adolfo Carit y Cleto González Víquez, con el aporte de su primer director: Marco Zúñiga (González, 1995). En relación con el tema, es destacable la relevancia de la enseñanza de la obstetricia para la fundación de la primera maternidad en el país, dato que permite concluir que la enfermería tuvo una función fundamental en el surgimiento de la estructura sanitaria dispuesta a servir a la enseñanza.

De acuerdo con Molina et al (2004), la apertura de la Escuela de Obstetricia significó un esfuerzo más de la profesión médica para subordinar ese saber popular, idea de la que se colige que el poder de dominio sobre la mujer se originaba en una cuestión de género de estos hombres con poder político, social y económico. El discurso es esperanzador para aquellas que tienen menos oportunidades, sin embargo, decidir qué y cómo enseñar significa un control que desestimaba importantes conocimientos de las parteras.

Algunos aspectos interesantes de la puesta en funcionamiento de la Escuela de Obstetricia, fue que para ser admitidas las alumnas debían ser mayores de 18 años y menores de 30 años, haber cursado la enseñanza primaria y gozar de buena salud y conducta. Además, en cada sección donde realizaran práctica iba a haber una hermana de la Caridad encargada del orden, la dieta, los medicamentos y el trabajo que debía realizar cada alumna. También, a partir del segundo año de carrera devengaron un sueldo de 15 colones mensuales como una ayuda para sus estudios (González, 1995).

La información presentada en relación con el tema es fundamental para esta investigación, para establecer elementos de análisis y destacar personajes clave, con el indagar sobre otras orientaciones posibles. Con los datos recabados se sienta los inicios, así como la precursora de la Enfermería profesional en Costa Rica, institución que en años posteriores se transformó en la primera Escuela de Enfermería del país.

\section{CONCLUSIONES}

Acerca de la presencia de la partería y el establecimiento de la enseñanza de la obstetricia en Costa Rica, se destaca algunas consideraciones preliminares:

Las condiciones de pobreza de la sociedad costarricense en el periodo en estudio , afectaron a una gran parte de la población, debido a la precaria atención de la salud, a lo que se suma la atención brindada en el hogar por la escasa existencia de centros sanitarios. En contraste, se habla de un grupo de mujeres adineradas, capaces de pagar los servicios que prestan los médicos, los cuales en su mayoría han estudiado en el extranjero.

La medicina tradicional y la curandería eran formas ampliamente utilizadas por la sociedad costarricense a finales del siglo XIX y principios del siglo XX, y se constituyeron en la base de la contradicción social entre las 
ideas liberales y la vida cotidiana costarricense, lo que motivó una especie de persecución desde las políticas públicas, implementadas principalmente por la profesión médica.

Hay una marcada diferencia de clases. Cuando se habla de establecer una Escuela de Obstetricia, se hace referencia a la atención de las mujeres de una Costa Rica muy pobre; sin embargo, cuando se habla de la posibilidad de que esta escuela sea sostenida económicamente por la caridad, se habla de mujeres que forman parte de una Costa Rica adinerada y próspera.

Aquí pueden generarse nuevas interrogantes que podrían ser profundizadas posteriormente, por ejemplo, ¿de qué manera la creación de la Escuela de Obstetricia en Costa Rica permitió mantener las diferencias de clase en las mujeres costarricenses? Las mujeres pobres ¿fueron atendidas de manera caritativa, mientras que las adineradas recibieron un trato particular de un médico de cabecera? ¿Contribuir a diferenciar la atención fue una función social que la Enfermería brindó a estas mujeres?

Se concluye que la fundación de la Escuela de Obstetricia se relacionó con las condiciones sociales, políticas y económicas de la época, basadas en la línea de desarrollo del país, caracterizadas por la necesidad de disminuir la tasa de mortalidad infantil y eliminar las prácticas populares de atención a la salud.

Por otra parte, la creación de la Escuela de Obstetricia, de acuerdo con lo encontrado en la Revista Gaceta Médica, dio paso a la construcción de una infraestructura hospitalaria para atender el parto que, a su vez, permitió el desarrollo de actividades académicas y de una nueva disciplina: la Enfermería.

\section{REFERENCIAS BIBLIOGRÁFICAS}

Borja, C. (1896). Escuela de Obstetricia. Revista Gaceta Médica de Costa Rica. 1 (4), 112.

Coelho, M., Susskind, M., Ballesteros, H. (2008). Investigación histórica en Enfermería. En do Horto, M., de Souza, M., Schmidt, K., Alonso, M. En do Prado, M., de Souza, M., Carraro, T. (2008). Investigación cualitativa en enfermería: contexto y bases conceptuales. Serie PALTEX Salud y Sociedad 2000 No. 9. Washington D.C.: OPS

Facultad de Medicina, Cirugía y Farmacia. (1900). Revista Gaceta Médica.

Facultad de Medicina, Cirugía y Farmacia. (1897). Varia. Beca. Revista Gaceta Médica 1(12). 351-352.

González, C. (2005). Memoria histórica de la Enfermería del Hospital San Juan de Dios. San José: Imprenta y Litografía Argentina

González, C. (1995). Hospital San Juan de Dios: 150 años de historia. San José: EDNASSS-CCSS

Gudmundson, L. (1993). Sociedad y Política (1840 - 1870). Por Pérez-Brignoli. (Ed.). Historia general de Centroamérica. De la Ilustración al liberalismo. Tomo III. Madrid: FLACSO-Sociedades Estatales Quinto Centenario-Ediciones Siruela 
Herrera, E. (1985). Los inmigrantes y el poder en Costa Rica. Revista de Historia. 11. Recuperado de revistas.una.ac.cr/index.php/historia/article/view/3185 [Consulta 15 de junio de 2104]

Junta de Caridad de San José. Informe: 1924-1925. Costa Rica: Alsina.

Junta de Caridad de San José. Informe: 1896. Costa Rica: Alsina.

Lachner, V. (1902). Apuntes de higiene pública. En: Revista de Costa Rica en el siglo XIX. Revista de Costa Rica en el Siglo XIX. Costa Rica: Imprenta Nacional.

Malavassi, A. (2002). De parteras a obstétricas: la profesionalización de una práctica popular. Costa Rica 1900-1940. En mujeres, género e historia. Costa Rica: UNIFEM

Marín, J. (1993). Las causas de la prostitución Josefina: 1939-1949. Entre lo imaginario y el estigma. Revista de Historia $N^{o}$ 27

Marín, J. (2000). De curanderos a médicos. Una aproximación a la historia social de la medicina en Costa Rica 1800-1949. Revista Electrónica de Historia. Vol, $1 \mathrm{~N}^{\circ} .2$

Molina, I., Palmer, S. (2004). La voluntad radiante. Cultura impresa, magia y medicina en Costa Rica (1897-1932). San José: EUNED

Ramírez, M. (1985). Campesinas, jornaleras, artesanas y amas de casa: el trabajo femenino rural en Costa Rica. Anuario de Estudios Centroamericanos 11 (1). 135-146.

Ros-Pochet (1896). Clínica Ginecológica. Revista Gaceta Médica de Costa Rica 1 (2), 51.

Rucavado, F. (1900) Nueva obra de beneficencia. Revista Gaceta Médica de Costa Rica, 5(4) 71.

Rosenberg, M. (1983). Las luchas por el seguro social en Costa Rica. San José: Editorial Costa Rica.

Salas J. (1987). La privatización de los baldíos nacionales en Costa Rica durante el siglo XIX. Legislación y procedimientos utilizados para su adjudicación. Revista de Historia. 15. 63-118.

Salazar, J. (1986). Estado liberal y luchas sociales. Revista de Ciencias Sociales, 92-93.

Samper, M. (1979). Evolución de la estructura socio-ocupacional costarricense: labradores, artesanos y jornaleros: 1864 1935. (Tesis para optar por la Licenciatura). Universidad de Costa Rica, Sede Rodrigo Facio.

Soto-Alfaro, J. (1896). Hidroanmios, hidrocéfalo. Revista Gaceta Médica de Costa Rica. 1(1), 5. 